\title{
A Case of Lung Cancer with a Patient with Situs Inversus Totalis
}

\author{
Alexei L. Charyshkin, $\mathrm{PhD}, \mathrm{ScD}^{1 *}$; Eugene A. Toneev ${ }^{1,2}$; Alexander A. Martynov'; \\ Dmitrij V. Bazarov, $\mathrm{PhD}^{3}$; Yuliya A. Dergunova ${ }^{1}$; Oleg V. Midlenko, $\mathrm{PhD}, \mathrm{ScD}^{1}$; \\ Antonina V. Smolkina, $\mathrm{PhD}, \mathrm{ScD}^{1}$; Nikolai I. Belonogov, $\mathrm{PhD}, \mathrm{ScD}^{1}$ \\ ${ }^{1}$ Institute of Medicine, Ecology and Physical Education of Ulyanovsk State University, Russia \\ ${ }^{2}$ Ulyanovsk Regional Oncology Center, Russia \\ ${ }^{3}$ Petrovsky Russian Research Center for Surgery, Russia
}

\begin{abstract}
In the current study, we describe a clinical case of the right upper lobectomy at Situs inversus totalis (SIT). Our report is the first described case in the domestic literature of SIT in a patient with multiple primary metachronous cancer, when a patient with SIT had primary lung cancer and successful surgical treatment. SIT is a complex and extremely rare clinical situation for the surgeon. In all such patients, it is advisable to use all available methods of imaging and diagnosis in the preoperative period, to clarify the anatomical structure of the vascular bed and bronchial tree. (International Journal of Biomedicine. 2019;9(2):176-178.)
\end{abstract}

Key Words: situs inversus totalis $\bullet$ lung cancer $\bullet$ right upper lobectomy

\section{Introduction}

Situs inversus totalis (SIT) — a completely reversed location of the internal organs - is a rare congenital autosomal recessive disorder that is associated with a defect on the $\mathrm{X}$ chromosome. The frequency of this pathology ranges from $1: 8000$ to $1: 20,000$ (1) $^{(1)}$ With this defect, an abnormal rotation of the heart tube occurs during embryogenesis. In $20 \%$ of cases, transposition is associated with the Kartagener syndrome, which includes a classical triad: situs inversus, sinusitis and bronchiectasis. ${ }^{(2)}$ Most people do not know about their peculiarities until they start to undergo various types of surveys. ${ }^{(3)}$ In the absence of functional disorders of the cardiovascular system, SIT can be attributed to normal variant anatomy, which does not have a significant impact on the quality of life. There are two main types of situs inversus. Dextrocardia with the normal position of the abdominal organs was first described in 1643 by Marco Severino.(4) SIT was described a century later, in 1797, by the British pathologist Matthew Baillie. ${ }^{(5)}$ There is also a variant with the position

*Corresponding author: Prof. Alexei L. Charyshkin, PhD, $S c D$, Head of the Faculty Surgery Department, Institute of Medicine, Ecology and Physical Education, Ulyanovsk State University. Ulyanovsk, the Russian Federation.E-mail: charyshkin@yandex.ru of the heart in the left half of the chest (levocardia) with transposition of the abdominal cavity, this phenomenon occurs in approximately 1:22,000 cases, and is called incomplete situs inversus. ${ }^{(6)}$ With the transposition of the internal organs with levocardia, heart defects are observed in $95 \%$ of cases..$^{(7)}$

Transposition of the internal organs with levocardia, or dextrocardia without transposition, are much more dangerous congenital defects than SIT. Dextrocardia (with the normal arrangement of the internal organs) is also found in some people with Patau syndrome (Trisomy 13). ${ }^{(8)}$

In the domestic literature, we did not find publications on anatomical resection of the lung in SIT patients. Considering all of the above, we considered it necessary to provide a description of the clinical case of the right upper lobectomy at SIT.

\section{Case presentation}

A 70-year-old man was admitted to the surgical thoracic department of our Clinical Hospital, complaining of a rare cough. The patient's medical history showed that in 2010, a total hemithyroidectomy was performed for a malignant disease of the thyroid gland. During the dynamic examination in December 2017, the progression of the process was revealed - cervical lymph node metastases, a tumor in the upper lobe of the right lung (Fig. 1-3).

In January 2018, a total thyroidectomy with cervical lymphadenectomy was performed. In a remote preparation, 
MTS of thyroid cancer were found. On 17 April 2018, radioiodine treatment was performed and a thoracic surgeon was recommended for radical treatment of the right lung tumor.

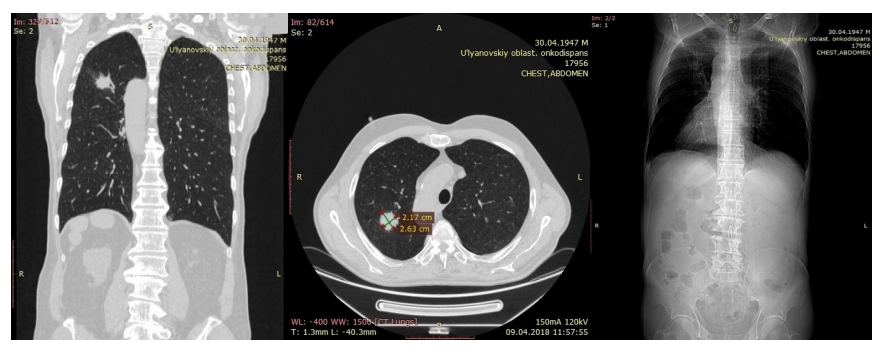

Fig. 1-3. MSCT: a tumor in the upper lobe of the right lung.

After the preoperative additional examination, the planned operation (05.31.2018) was performed: Right thoracotomy, upper lobectomy, systemic lymph node dissection (Fig.4). Intraoperatively: the right-sided position of the heart and aorta. In the upper lobe of the right lung, a formation with a contraction of the pleura was detected $(3 \times 3$ $\mathrm{cm}$ in size), mediastinal lymph nodes were not enlarged. A standard upper lobectomy with systemic lymph node dissection was performed. The postoperative period was uneventful, the wound healed by first intention. Drains were removed on the third day. The duration of the operation was 96 minutes, the blood loss was minimal.

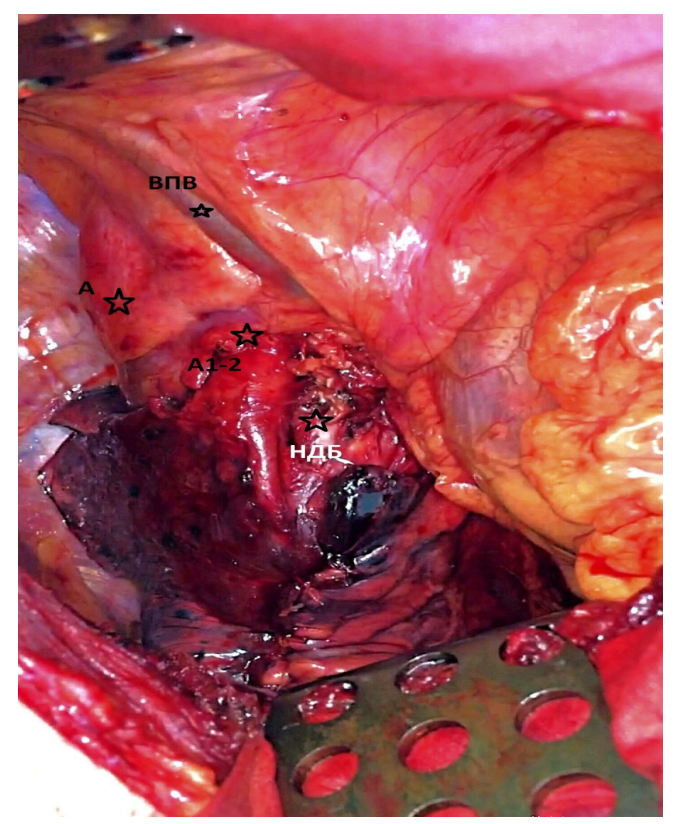

Fig. 4. Intraoperatively photo (A - aorta, A1-2segmental artery, НДБ- lower lobe bronchus).

Histological examination: papillary cancer (morphology characteristic of thyroid cancer and primary lung adenocarcinoma). All groups of lymph nodes of the lung root and mediastinum were without a tumor lesion.

Immunohistochemical examination: TTF1-positive reaction; CDX2, Tireoglobulin, CK20-negative reaction. Given the immunophenotype and morphology, more data are available for primary papillary adenocarcinoma of the lung (pT1aN0R0) (Figure 5-7.)

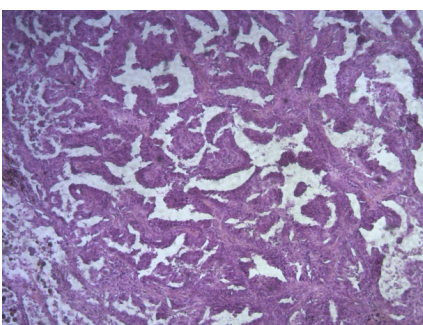

Fig. 5. Primary papillary adenocarcinoma. $H \& E, \times 100$

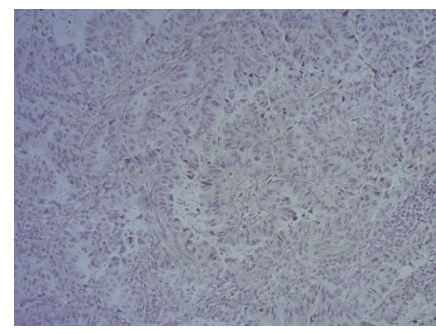

Fig. 7. Immunohistochemistry: $T g$ Ab-negative result, $\times 200$
Thus, the clinical diagnosis: Multiple primary metachronous cancer.

1. Thyroid cancer (pT2N0M0), operation 2010. Progression of 12.2017-MTS to cervical lymph nodes, operation in 01.2018 .

2. Lung cancer of the right upper lobe (pT1aNOM0).

\section{Discussion}

Most of the published papers on surgical intervention in patients with SIT are presented mainly about the abdominal organs (cholecystectomy, appendectomy). ${ }^{(9)}$ There are isolated reports of SIT cases during operations on the colon, ${ }^{(10)}$ stomach, ${ }^{(11)}$ esophagus ${ }^{(12)}$ and the heart; ${ }^{(13)}$ there is a publication about lung transplantation. ${ }^{(14)}$ Currently, the presence of a solitary neoplasm in the lung is an indication for its anatomical resection. It should be borne in mind that the presence of SIT does not guarantee the standard vascular and bronchial anatomy ${ }^{(15)}$ When planning an operation in patients with SIT, the available vascular or bronchial anatomical features should always be taken into account at the preoperative stage, which promotes a safe surgery. In our case, the preoperative MSCT showed the presence of two main arterial trunks going to the upper lobe: A1-2 and A3,4,5. Endoscopic ultrasonography (EUS) and endobronchial ultrasonography (EBUS) can be used as additional methods for specifying diagnosis. ${ }^{(16)}$

Subotich et al. considered that preoperative angiography should be performed on all patients with SIT to study the vascular anatomy of the lung, due to the high possibility of the presence of vascular anomalies. ${ }^{(17)}$ We believe that a 3D diagnostic CT and MRI will be useful in a diagnostic search. 
In the present case, MSCT was sufficient to obtain a complete picture of the vascular bed and the bronchial tree.

Our report is the first described case in the domestic literature of SIT in a patient with multiple primary metachronous cancer and is the 29th case in the available literature since 1952, when a patient with SIT had primary lung cancer and successful surgical treatment.

SIT is a complex and extremely rare clinical situation for the surgeon. It is necessary to know the patient's exact topographic anatomy, which will avoid intraoperative complications. In all such patients, it is advisable to use all available methods of imaging and diagnosis in the preoperative period, to clarify the anatomical structure of the vascular bed and bronchial tree.

\section{Competing Interests}

The authors declare that they have no competing interests.

\section{References}

1. Wójcik J, Grodzki T, Bielewicz M, Wojtyś M, Kubisa B, Pieróg J, Wójcik N. Lung cancer in situs inversus totalis (SIT)--literature review. Adv Med Sci. 2013;58(1):1-8. doi: 10.2478/v10039-012-0083-x.

2. Reimer TV, Mursalimova VF, Meteleva AV. [Family case of Kartagener syndrome]. Vestnik Orenburg State University. 2011;16(135):331-2. [Article in Russian].

3. Bondarenko IA, Chesnakova TV. [Situs Viscerus Inversus Totalis (Clinical Case)]. Radiology — Practice. 2014;(2):5763. [Article in Russian].

4. Maria Conforti. Surgery, medicine and natural philosophy in the library of Marco Aurelio Severino (1580-1956). Bruniana \& Campanelliana.2004;10(2):283-298.

5. Kaleda VI, Glyantsev SP. [Transposition of the great arteries: The first description by M. Baillie in 1797]. Heart and Vessels Diseases in Children. 2010;(4): 51-55. [Article in Russian].

6. Wang X, Shi Y, Zeng S, Zhou J, Zhou J, Yuan H, et al. Comparing levocardia and dextrocardia in fetuses with heterotaxy syndrome: prenatal features, clinical significance and outcomes. BMC Pregnancy Childbirth. 2017;17(1):393. doi: 10.1186/s12884-017-1579-y.
7. Del Prete A, Cavaliere C, Di Pietto F, De Ritis R. Situs inversus with levocardia, infrahepatic interruption of the inferior vena cava, and azygos continuation: a case report. Surg Radiol Anat. 2016;38(3):369-71. doi: 10.1007/s00276015-1454-3.

8. Gainazarova AS, Ibragimova TM, Kaliev RR. [Clinical case of a combination of dextrocardia and cardiac arrhythmias]. Bulletin of KSM A named after I.K. Akhunbaev. 2016;(4):3537. [Article in Russian].

9. Tacchino R, Greco F, Matera D. Single-incision laparoscopic cholecystectomy: surgery without a visible scar. Surg Endosc. 2009;23(4):896-9. doi: 10.1007/s00464-008-0147-y.

10. Kim HJ, Choi GS, Park JS, Lim KH, Jang YS, Park SY, Jun SH. Laparoscopic right hemicolectomy with D3 lymph node dissection for a patient with situs inversus totalis: report of a case. Surg Today. 2011;41(11):1538-42. doi: 10.1007/ s00595-010-4530-7.

11. Dibirov MD, Fomin VS. [Laparoscopy-assisted gastrectomy for gastric carcinoma in a patient with complete situs inversus.]. Moscow Surgical Journal. 2017;53(1):34-37. [Article in Russian]. 12. Yoshida T, Usui S, Inoue H, Kudo SE. The management of esophageal cancer with situs inversus totalis by simultaneous hand-assisted laparoscopic gastric mobilization and thoracoscopic esophagectomy. J Laparoendosc Adv Surg Tech A. 2004;14(6):384-9.

13. Iino $\mathrm{K}$, Watanabe $\mathrm{G}$, Ishikawa $\mathrm{N}$, Tomita $\mathrm{S}$. Total endoscopic robotic atrial septal defect repair in a patient with dextrocardia and situs inversus totalis. Interact Cardiovasc Thorac Surg. 2012;14(4):476-7. doi: 10.1093/icvts/ivr103.

14. Macchiarini P, Chapelier A, Vouhé P, Cerrina J, Ladurie FL, Parquin $\mathrm{F}$, et al. Double lung transplantation in situs inversus with Kartagener's syndrome. Paris-Sud University Lung Transplant Group. J Thorac Cardiovasc Surg. 1994;108(1):86-91.

15. Safdie FM, Sanchez MV, Sarkaria IS. Prevention and management of intraoperative crisis in VATS and open chest surgery: how to avoid emergency conversion. J Vis Surg. 2017;3:87. doi: 10.21037/jovs.2017.05.02.

16. Yoshida M, Hino H, Machida H, Hatakeyama N, Okano $\mathrm{Y}$, Iwahara Y, et al. Video-assisted thoracic surgery lobectomy for lung cancer in a patient with complete situs inversus. Gen Thorac Cardiovasc Surg. 2013;61(3):155-9. doi: 10.1007/ s11748-012-0114-3.

17. SubotichD, MandarichD, KatcharV, Bulajich B, Drndarski $B$. Lung resection for primary bronchial carcinoma in a patient with complete situs inversus. Clin Anat. 2006;19(4):358-62. 